DDS - Die Deutsche Schule

113. Jahrgang 2021, Heft 1, S. 85-100

https://doi.org/10.31244/dds.2021.01.08

CC BY-NC-ND 4.0 Waxmann 2021

Habibe Ercan, Ulrike Hartmann, Dirk Richter, Jenny Kuschel

\& Cornelia Gräsel

\title{
Effekte von integrativer Führung auf die Datennutzung von Lehrkräften
}

\section{Zusammenfassung}

Die vorliegende Studie untersucht die Zusammenhänge zwischen integrativem Schulleitungshandeln, das transformationale und instruktionale Komponenten enthäl, und der Nutzungshäufigkeit verschiedener Datenquellen durch Lehrkräfte. Die Ergebnisse eines Strukturgleichungsmodells zeigen, dass integrative Führung direkte und indirekte Zusammenhänge mit der Nutzung verschiedener Datenquellen aufweist. Die Effekte scheinen vorwiegend durch die Kooperationsaktivität der Lehrkräfte vermittelt zu sein. Schlüsselwörter: Datennutzung von Lehrkräften, integrative Führung, Kooperation

\section{Data-based School Improvement - Direct and Indirect Effects of Integrative Leadership on Teachers' Data Use}

\begin{abstract}
The present article reports the effects of school principals' integrative leadership behavior, including transformational and instructional components, on teachers' data use. Results of a structural equation model indicate significant positive direct and indirect effects on teachers' use of various data. Total effects seem to be mainly mediated by teachers' cooperation activities.
\end{abstract}

Keywords: teachers' data use, integrative leadership, cooperation

\section{$1 \quad$ Einleitung}

Datengestützte Schul- und Unterrichtsentwicklung gewinnt in der Schulentwicklung zunehmend an Bedeutung. Schulleitungen und Lehrkräfte sollten in der Lage sein, geeignete Daten sowie wissenschaftliche Resultate für pädagogische Entscheidungen zu kennen und heranzuziehen, um daraus Konsequenzen für die Schul- und Unter- 
richtsentwicklung abzuleiten (z. B. Bromme, Prenzel \& Jäger, 2014; Thiel, Hannover \& Pant, 2014); der Prozess entspricht der datengestützten Entscheidungsfindung (DataDriven-Decision-Making, DDDM). Die Nutzung empirischer Daten kann somit als eine Schnittstelle der medial vermittelten Kommunikation zwischen Einzelschule und Wissenschaft betrachtet werden.

Daten werden in der vorliegenden Studie als systematisch gesammelte und geordnete Informationen verstanden, die schulische Aspekte widerspiegeln (Schildkamp \& Lai, 2013). Sie können sowohl qualitativ als auch quantitativ gesammelt, innerschulisch (z. B. Schüler^innennoten) als auch außerschulisch (z. B. Schulinspektionsergebnisse) generiert sowie formeller (z.B. Fachliteratur) oder informeller Art (z.B. allgemeine Medien) sein. Alle Quellen können genutzt werden, um pädagogisch relevante Entscheidungen datengestützt zu treffen (Ikemoto \& Marsh, 2007) und die Schulund Unterrichtsqualität $\mathrm{zu}$ erhöhen. Diese Kompetenz von Lehrkräften wird in der US-amerikanischen Bildungsforschung als data literacy zusammengefasst (z.B. Mandinach \& Gummer, 2016; Schildkamp, Poortman, Luyten \& Ebbeler, 2017).

Zahlreiche Befunde zeigen, dass sich das Ziel einer datengestützten Schulentwicklung nicht ohne Schwierigkeiten umsetzen lässt (Zlatkin-Troitschanskaia et al., 2016). $\mathrm{Ob}$ und wie Lehrpersonen datenbasiert Entscheidungen treffen, ist von multiplen Faktoren und deren Interaktionen abhängig. Insbesondere wird den persönlichen Merkmalen von Lehrkräften (z. B. ihren Kompetenzen und Haltungen; Schildkamp \& Lai, 2013; Albiladi, Lasater \& Bengtson, 2020), den Handlungsroutinen und Merkmalen der Schulorganisation eine wichtige Bedeutung beigemessen (z.B. Stump, Zlatkin-Troitschanskaia \& Mater, 2016; Coburn \& Turner, 2011; Anderson, Leithwood \& Strauss, 2010). Als eine wichtige förderliche Einflussgröße für datenbasiertes Entscheiden wird eine kooperative Schulkultur beschrieben (Altrichter, 2010; Schildkamp \& Ehren, 2013). Eine enge Kooperation von Lehrkräften - für die die entsprechenden zeitlichen und räumlichen Voraussetzungen bestehen - unterstützt den Austausch und die Diskussion über Daten und insbesondere das Ableiten von Konsequenzen für die Schul- und Unterrichtsentwicklung (Schildkamp et al., 2017).

Schulleitungen nehmen für die datengestützte Schul- und Unterrichtsentwicklung damit eine besonders wichtige Rolle ein: Durch ihr Führungsverhalten beeinflussen sie sowohl die Voraussetzungen der Lehrkräfte (Lewin \& Datnow, 2012) als auch die organisationalen Bedingungen, insbesondere für die Zusammenarbeit (vgl. Datnow \& Hubbard, 2016). Sie können strukturelle Rahmenbedingungen und Zeit schaffen, sodass Lehrkräften geeignete Daten zur Verfügung stehen und sie die Möglichkeit haben, sich mit diesen intensiv auseinanderzusetzen. Schulleitungen gewährleisten auch den Aufbau innerschulischer Kooperationsstrukturen und damit den kollegialen Austausch über Daten. Schließlich wird die Datennutzung durch Lehrkräfte positiv beeinflusst, wenn verbindliche Regeln zum Umgang mit Daten aufgestellt werden (Marsh, 2012) und die Schulleitung als Experte für Datennutzungsfragen zur 
Verfügung steht (z.B. Ikemoto \& Marsh, 2007). Erste empirische Studien geben Hinweise darauf, dass für die Implementierung des datenbasierten Handelns in schulische Prozesse das Schulleitungshandeln von Bedeutung ist (Brown \& Zhang, 2016; Leithwood \& Sun, 2012).

\section{Führung an Schulen - ein integrativer Ansatz}

Für die Analyse von Schulleitungshandeln wurden in der Schuleffektivitätsforschung zwei Führungskonzepte herausgearbeitet, nämlich das transformationale und das instruktionale. Hinsichtlich einer erfolgreichen Schulentwicklung wird eine ausgeglichene Kombination dieser Konzepte als funktional angenommen (Heck \& Hallinger, 2010; Pietsch, 2014). An dieser Integration orientiert sich auch dieser Aufsatz.

Transformationale Führung ist gekennzeichnet durch eine starke Mitarbeiter ${ }^{\star}$ innenorientierung, die direkte Formulierung überzeugender und attraktiver Zukunftsvisionen für die Schule, die Inspiration und Motivation der Lehrkräfte sowie die Fähigkeit, deren Bedürfnisse zu erkennen, individuelle Stärken und kollektive Selbstwirksamkeit $\mathrm{zu}$ fördern. Bisherige Befunde zeigen, dass sich transformationales Schulleitungshandeln, direkt sowie vermittelt über motivationale Faktoren und die Stärkung kollegialer Kooperation, auf die Bereitschaft von Lehrkräften zur Datennutzung auswirkt (z. B. Stump et al., 2016; Pietsch \& Hosenfeld, 2017). Instruktionale (auch pädagogische) Führung umfasst vor allem die Verbesserung der lehr- und lernrelevanten Strukturen und Prozesse an einer Schule. Die Schulleitung nimmt dabei gezielt Einfluss auf die Unterrichtsgestaltung, koordiniert Lehrpläne und steht als kompetente ${ }^{\star} r$ Ansprechpartner*in bei Problemen in Unterrichtsfragen zur Verfügung (Hallinger, Leithwood \& Heck, 2010). Damit können Aspekte dieses Führungsstils das Ableiten von Konsequenzen aus Daten sowie die konkrete Implementierung der Konsequenzen in den Schulalltag unterstützen. Pietsch und Hosenfeld (2017) konnten auch für ein instruktionales Führungsverhalten direkte Effekte auf die datengesteuerte Unterrichtsentwicklung nachweisen.

Aufgrund dieser theoretischen Überlegungen und bisheriger empirischer Ergebnisse gehen wir davon aus, dass für die datenbasierte Schul- und Unterrichtsentwicklung eine Integration beider Führungskonzeptionen besonders unterstützend ist. Die Vorgabe motivierender Leitbilder sowie das Erkennen der Entwicklungsbedarfe (im Sinne transformationaler Führung) und das Monitoring von Lehr- und Lernprozessen (im Sinne instruktionaler Führung) scheinen wichtige Wirkmechanismen zu sein. Zu einem entsprechenden integrativen Ansatz gehören die Kommunikation der schulischen Zielvorstellungen, die Verfügbarkeit als kompetente ${ }^{\star} r$ Ansprechpartner ${ }^{\star}$ in, die Schaffung günstiger struktureller Arbeitsbedingungen sowie eine umfassende Ressourcen- und Problemlösungsorientierung. Im vorliegenden Beitrag wird unter- 
sucht, welcher Zusammenhang zwischen einer integrativen Führung der Schulleitung und der Nutzung einzelner schulischer Datenquellen durch Lehrkräfte besteht. Außerdem werden die für die Datennutzung bedeutsamen Kooperationsaktivitäten (Altrichter, 2010; Schildkamp \& Ehren, 2013) sowie die Einstellung von Lehrkräften gegenüber einer datenbasierten Schulpraxis (Schildkamp \& Lai, 2013) berücksichtigt.

Welche Datenquellen die Lehrkräfte nutzen, ist bislang kaum erforscht. Empirische Einzelbefunde machen deutlich, dass die einfache Verfügbarkeit von Informationen, die von Lehrkräften wahrgenommene Reliabilität und Validität der Daten sowie die zeitliche Nähe (von Rückmeldungen) eine entscheidende Rolle für die Nutzungsintensität spielen (Ikemoto \& Marsh, 2007; Anderson et al., 2010). Um mehr Erkenntnis darüber zu gewinnen, welche Quellen Lehrkräfte nutzen, verwendet die folgende Untersuchung einen sehr breiten „Datenbegriff“ (vgl. Tab. 3 auf S. 92). ${ }^{1}$

\section{Fragestellungen der Studie}

1) Wie hoch ist die Nutzungshäufigkeit von einzelnen Datenquellen, die Lehrkräften zur Verfügung stehen?

2) Welche direkten und indirekten Zusammenhänge (vermittelt über Kooperationsaktivitäten und Haltungen gegenüber einer datengestützten Schul- und Unterrichtsentwicklung der Lehrpersonen) bestehen zwischen einer integrativen Führung der Schulleitung und der Datennutzung von Lehrkräften?

\section{Methode}

\subsection{Datengrundlage und Stichprobe}

Die Daten stammen aus dem Projekt „CoMMIT - Kooperation an Schulen, Innovation im Team“, welches durch die Robert-Bosch-Stiftung und Die Deutsche Schulakademie gefördert wurde. ${ }^{2}$ Das Projekt wird mit insgesamt 16 freiwillig teilnehmenden weiterführenden Schulen aus sechs Bundesländern durchgeführt. Die Grundlage für die vorliegende Untersuchung beruht auf Daten der Eingangsbefragung von $N=776$ Lehrkräften (davon 61,5\% weiblich; Durchschnittsalter: 42,8 Jahre). Diese wurde im Rahmen von Lehrer*innenkonferenzen mit einem Paper-and-Pencil-

1 In der Literatur zur Nutzung von Forschungsergebnissen ist generell eine unklare Definition bzw. Abgrenzung festzustellen, was „Daten“ und was „Forschungsergebnisse“ sind (vgl. Hinzke, Gesang \& Besa, 2020). Wir haben uns gegen den Begriff der „Evidenz(en)“ entschieden, weil dieser gerade in erziehungswissenschaftlichen Kontexten zu (zum Teil nicht nachvollziehbaren) emotionalen Debatten führt, und verwenden deswegen das neutralere „Daten“ in einem breiten Sinne.

2 Nähere Informationen zum Projekt können der Homepage https://www.ifb.uni-wuppertal. de/en/institut/projekte/projekt-commit.html entnommen werden. 
Fragebogen durchgeführt. Durchschnittlich nahmen 70 Prozent der Lehrkräfte eines Kollegiums an der Befragung teil.

\subsection{Variablen und Messinstrumente}

Aspekte der integrativen Führung der Schulleitungen wurden mit zehn Items erfasst (vierstufige Likert-Skala; $1=$ trifft nicht $\mathrm{zu} ; 4=$ trifft voll $\mathrm{zu}$ ). Items zur Erfassung der transformationalen Aspekte (z. B. „Die Schulleitung schafft es, unseren Teamgeist durch überzeugende Zukunftsvisionen zu stärken.") wurden in Anlehnung an Skalen aus dem Multifactor Leadership Questionnaire (MLQ; vgl. Bass \& Avolio, 1994) entwickelt. Items für instruktionale Verhaltensweisen (z. B. „Die Schulleitung steht bei der Umsetzung von Zielvorgaben an der Schule als kompetenter Ansprechpartner zur Verfügung.") wurden anhand der Skalen aus dem Teaching and Learning International Survey (TALIS; vgl. Schmich \& Schreiner, 2008) entwickelt. Eine konfirmatorische Faktorenanalyse (CFA) für ein einfaktorielles Faktorenmodell weist eine gute Passung auf $\left(\chi^{2}=207.249 ; \quad \mathrm{df}=35 ; p<.001 ; \quad \mathrm{CFI}=.96 ; \quad \mathrm{TLI}=.95 ; \quad \mathrm{RMSEA}=.082 \quad(90 \%\right.$ $\mathrm{CI}=.072 / .093) ; \mathrm{SRMR}=.029)^{3}$. Alle Faktorladungen der zehn Items sind signifikant und liegen zwischen .70 und .83 (vgl. Tab. 1 auf der folgenden Seite). Auch die hohen Inter-Item-Korrelationen sprechen dafür, dass die verwendeten Items ein eindimensionales Führungskonzept abbilden und Schulleitungen sich lediglich in dessen Ausmaß unterscheiden. Die interne Konsistenz der Skala liegt bei $\alpha=.91$.

Haltungen gegenüber der datengestützten Schul- und Unterrichtsentwicklung der Lehrkräfte wurden mittels sechs Items der Evidence-Based Practice Attitude Scale (EBPAS; vgl. Aarons et al., 2010) erhoben (vierstufige Skala; 1 = stimme nicht zu; 4 = stimme vollkommen zu; Beispielitem: „Mir ist es wichtig, dass meine pädagogischen Handlungen mit aktuellen wissenschaftlichen Erkenntnissen zusammenpassen. “; $\alpha=.78)$.

Die Kooperationsaktivitäten der Lehrkräfte wurden mit einem Vier-Faktorenmodell von Kooperationsformen (Austausch, Kokonstruktion) und -inhalten (unterrichtsbezogen, schulbezogen) operationalisiert (Hartmann, Richter \& Gräsel, 2020; vgl. Tab. 2 auf S. 91). Vorgegebene Kooperationsaktivitäten wurden auf einer vierstufigen Skala beantwortet ( 1 = nie; 2 = selten; 3 = gelegentlich; 4 = häufig). Die Skalen Unterrichtsbezogener Austausch (Beispielitem: "Ich tausche Unterrichtsmaterialien ( $z$. B. Aufgaben und Arbeitsblätter) mit Kolleginnen und Kollegen aus."), Unterrichtsbezogene Kokonstruktion (Beispielitem: „Ich diskutiere mit Kolleginnen und Kollegen über meine Unterrichtsentwürfe mit dem Ziel, diese zu optimieren."), Schulbezogener Austausch

3 Getestete mehrfaktorielle Modelle besitzen maximal vergleichbar gute Modellgüteparameter. Aufgrund der theoretischen Annahme einer umfassenden, integrativen Führungskonzeption wird ein einfaktorielles Modell herangezogen. Statistisch wird dieses Modell durch die hohen Inter-Item-Korrelationen bekräftigt. 
(Beispielitem: „Ich berichte Kolleginnen und Kollegen, wenn ich etwas Neues gelernt habe (z. B. bei Fortbildungen, durch Lektüre).") sowie die Skala Schulbezogene Kokonstruktion (Beispielitem: „Ich spreche mich mit Kolleginnen und Kollegen darüber ab, wie wir das Schulprogramm in unserem Unterricht umsetzen.") weisen knapp zufriedenstellende Reliabilitäten auf (Cronbachs Alpha zwischen .58 und .66), und konfirmatorische Faktorenanalysen stützen diese Einteilung. Jede Skala wird durch drei Items abgebildet.

Tab. 1: Items zur Erfassung des integrativen Führungsstils von Schulleitungen und dazugehörige Faktorladungen

\section{Item}

a) Wenn es Probleme innerhalb des Kollegiums gibt, gibt die Schulleitung uns Freiräume, dafür neue Lösungen zu finden.

b) Die Schulleitung ermuntert uns darin, unsere Lehr- und Lernpraktiken selbstständig weiterzuentwickeln.

c) Die Schulleitung schafft es durch ihre Begeisterung, die Ziel- und Wertorientierungen der Schule nachhaltig zu verändern.

d) Meine individuellen Potenziale werden von der Schulleitung erkannt und gefördert.

e) Die Schulleitung steht bei der Umsetzung von Zielvorgaben an der Schule als kompetenter Ansprechpartner zur Verfügung.

f) Die Schulleitung schafft im Kollegium Bedingungen zur Bewältigung schulischer und außerschulischer Probleme.

g) Die Schulleitung sorgt dafür, dass Probleme innerhalb des Kollegiums zeitnah geklärt werden.

h) Die Schulleitung schafft es, unseren Teamgeist durch überzeugende Zukunftsvisionen zu stärken.

i) Die Schulleitung stellt für mich ein Vorbild für gutes pädagogisches Handeln dar.

j) Die Schulleitung informiert uns darüber, wie sie neue Vorgaben in der Weiterentwicklung unseres Schulprofils berücksichtigt.

\section{Faktorladung}

$0.72^{*}$

$0.70^{*}$

$0.79 *$

$0.73^{*}$

$0.82^{*}$

$0.80^{*}$

$0.76^{*}$

$0.82 *$

$0.83^{*}$

$0.73^{*}$

Anm.: Signifikanzniveau: ${ }^{*}<.05$

Quelle: eigene Berechnungen 
Effekte von integrativer Führung auf die Datennutzung von Lehrkräften

Tab. 2: Mittelwerte, Standardabweichungen, Itemanzahl und Anzahl der berücksichtigten Fälle für die eingesetzten Skalen

\begin{tabular}{lcccc}
\hline \multicolumn{1}{c}{ Skala } & $\begin{array}{c}\text { Anzahl der } \\
\text { Items }\end{array}$ & $\boldsymbol{M}$ & SD & $\boldsymbol{n}$ \\
\hline Integrative Führung & 10 & 2.77 & 0.73 & 585 \\
$\begin{array}{l}\text { Kooperationsverhalten } \\
\quad \begin{array}{l}\text { Unterrichtsbezogener } \\
\text { Austausch }\end{array}\end{array}$ & 12 & 2.72 & 0.70 & 707 \\
$\begin{array}{l}\text { Unterrichtsbezogene } \\
\text { Kokonstruktion }\end{array}$ & 3 & 3.29 & 0.53 & 760 \\
$\begin{array}{l}\text { Schulbezogener } \\
\text { Austausch }\end{array}$ & 3 & 2.11 & 0.86 & 745 \\
$\begin{array}{l}\text { Schulbezogene } \\
\text { Kokonstruktion }\end{array}$ & 3 & 2.82 & 0.68 & 740 \\
$\begin{array}{l}\text { Einstellung zu evidenz- } \\
\text { basierter Praxis }\end{array}$ & 6 & 2.75 & 0.74 & 740 \\
\hline
\end{tabular}

Anm.: $\quad M=$ Mittelwert, $S D=$ Standardabweichung, $n=$ berücksichtigte Fälle pro Skala.

Quelle: eigene Berechnungen

Als abhängige Variable wurde die Nutzungshäufigkeit von insgesamt zwölf Formen von Daten- und Informationsquellen herangezogen. Die Lehrkräfte wurden gebeten, auf einer vierstufigen Skala ( 1 = nie, 2 = selten; 3 = gelegentlich; $4=$ häufig $)^{4}$ für jede der zwölf Informationsquellen folgende Frage zu beantworten: „Bitte geben Sie an, wie oft Sie folgende Informationsquellen für Ihre Arbeit nutzen. "Eine Übersicht der abgefragten Quellen liefert Tabelle 3 in Kapitel 5.1.

\subsection{Analysen}

Zur Beantwortung der Forschungsfragen wurden deskriptive Statistiken berichtet und ein Strukturgleichungsmodell mit dem Maximum-Likelihood-Verfahren geschätzt (mit MPLUS 7.0; vgl. Muthén \& Muthén, 2006). ${ }^{5}$ Dabei ging es um die Passung eines Modells zu direkten und indirekten Effekten einer integrativen Führung auf die Nutzungshäufigkeit von Daten- und Informationsquellen durch Lehrkräfte. Als Gütekriterien für die Modellanpassung werden der Comparative Fit Index (CFI), der

4 Wie nicht nur in der Kooperationsforschung, sondern in vielen Arbeiten der Schul- und Unterrichtsforschung üblich, wurden alle zeitlich gradierenden Skalen als intervallskaliert ausgewertet.

5 Aufgrund der relativ kleinen Anzahl an Teilnehmerschulen und der eher geringen Intraklassenkorrelationen der verwendeten Items haben wir auf eine Mehrebenenmodellierung der Daten verzichtet (in der Regel werden ICC-Werte von $\geq 0,7$ für die Aggregation von Individualdaten auf höheren Ebenen eingefordert; vgl. Lüdtke, Trautwein, Kunter \& Baumert, 2006. Somit ist die statistische Notwendigkeit einer Mehrebenenkonstruktion nicht gegeben). 
Tucker Lewis Index (TLI), der Root Mean Square Error of Approximation (RMSEA) sowie der Standardized Root Mean Square Residual (SRMR) berichtet. Als akzeptable Modell-Fits gelten dabei folgende Werte (Marsh, Hau \& Wen, 2004): CFI- und TLIWerte $\geq .90$, RMSEA-Werte $\leq .08$ und SRMR-Werte $\leq .08$. Fehlende Werte wurden mit der Maximum-Likelihood-Schätzung behandelt.

\section{Ergebnisse}

\subsection{Ergebnisse zur Nutzung der abgefragten Datenquellen}

Tabelle 3 zeigt, dass es große Unterschiede in der Nutzungshäufigkeit der abgefragten Daten- und Informationsquellen gibt. Im Vergleich $\mathrm{zu}$ anderen Quellen fällt die Nutzung von allgemeinen Medien (z.B. Tageszeitungen, Internet) am größten aus. Für weitere, insbesondere schüler*innenbezogene Quellen wie z.B. Fehlzeiten gaben Lehrkräfte an, dass sie diese selten bis gelegentlich nutzen. Die Nutzung von schulinternen Daten (z.B. Evaluationen) und bildungswissenschaftlichen Forschungsresultaten (z. B. aus pädagogischen oder psychologischen Datenbanken) fällt geringer aus. Abschlüsse von Abschlussjahrgängen, Daten aus Vergleichsarbeiten und Ergebnisse der Schulinspektion werden noch seltener genutzt.

Tab. 3: Mittelwerte und Standardabweichungen der abgefragten Daten- und Informationsquellen (Lehrkräftebefragung) sortiert nach dem Umfang der Nutzung

\begin{tabular}{lcc}
\hline \multirow{2}{*}{ Daten- und Informationsquellen } & \multicolumn{2}{c}{ Nutzungshäufigkeit } \\
\hline Allgemeine Medien (z.B. Tageszeitung, Internet) & $M$ & $S D$ \\
Schüler*innenfehlzeiten & 3.63 & 0.64 \\
Noten einzelner Schüler*innen & 2.93 & 0.99 \\
Schüler*innenfeedback zum Unterricht & 2.86 & 0.95 \\
Fachliteratur & 2.77 & 1.02 \\
Unterrichtsausfall/Krankenstand & 2.58 & 0.94 \\
Schulinterne Evaluation & 2.26 & 1.16 \\
Befragungen von Schüler*innen und Eltern & 1.97 & 9.93 \\
Bildungswissenschaftliche Forschungsresultate & 1.93 & 0.98 \\
Erreichte Abschlüsse des Abschlussjahrgangs & 1.86 & 0.91 \\
Daten aus Vergleichsarbeiten & 1.77 & 0.96 \\
Ergebnisse aus der Schulinspektion & 1.76 & 0.96 \\
\hline
\end{tabular}

Anm.: $\quad M=$ Mittelwert, $S D=$ Standardabweichungen.

Quelle: eigene Berechnungen 


\subsection{Zusammenhänge zwischen integrativem Schulleitungshandeln und der Datennutzung von Lehrkräften}

Für die Beantwortung der zweiten Fragestellung wurde ein Strukturgleichungsmodell geschätzt (vgl. Abb. 1 und Tab. 4 auf S. 95); die Fit-Indices sind zufriedenstellend $\left(\chi^{2}=1319.415 ; \mathrm{df}=644 ; p<.001 ; \mathrm{CFI}=.92 ; \mathrm{TLI}=.91 ; \mathrm{RMSEA}=037(90 \%\right.$ $\mathrm{CI}=.034 / .040)$; SRMR $=.047)$. Anhand der Modellvariablen lassen sich etwa fünf bis 20 Prozent der Gesamtvarianz in der Nutzungshäufigkeit der einzelnen Datenquellen erklären. Die größte Varianzaufklärung der integrativen Führung besteht bei der Nutzung schulinterner Evaluationen und bildungswissenschaftlicher Forschungsresultate sowie bei der Nutzung von Befragungen von Schüler*innen und Eltern und Schulinspektionsergebnissen. Weiterhin zeigen die Ergebnisse, dass integrative Führung positiv mit der Kooperationsaktivität der Lehrkräfte und der positiven Haltung von Lehrkräften gegenüber der datenbasierten Schul- und Unterrichtsentwicklung zusammenhängt.

Abb. 1: Darstellung des Strukturgleichungsmodells zur Beschreibung der direkten und indirekten Effekte von integrativer Führung auf die Nutzungshäufigkeit von Datenquellen 1 bis 12

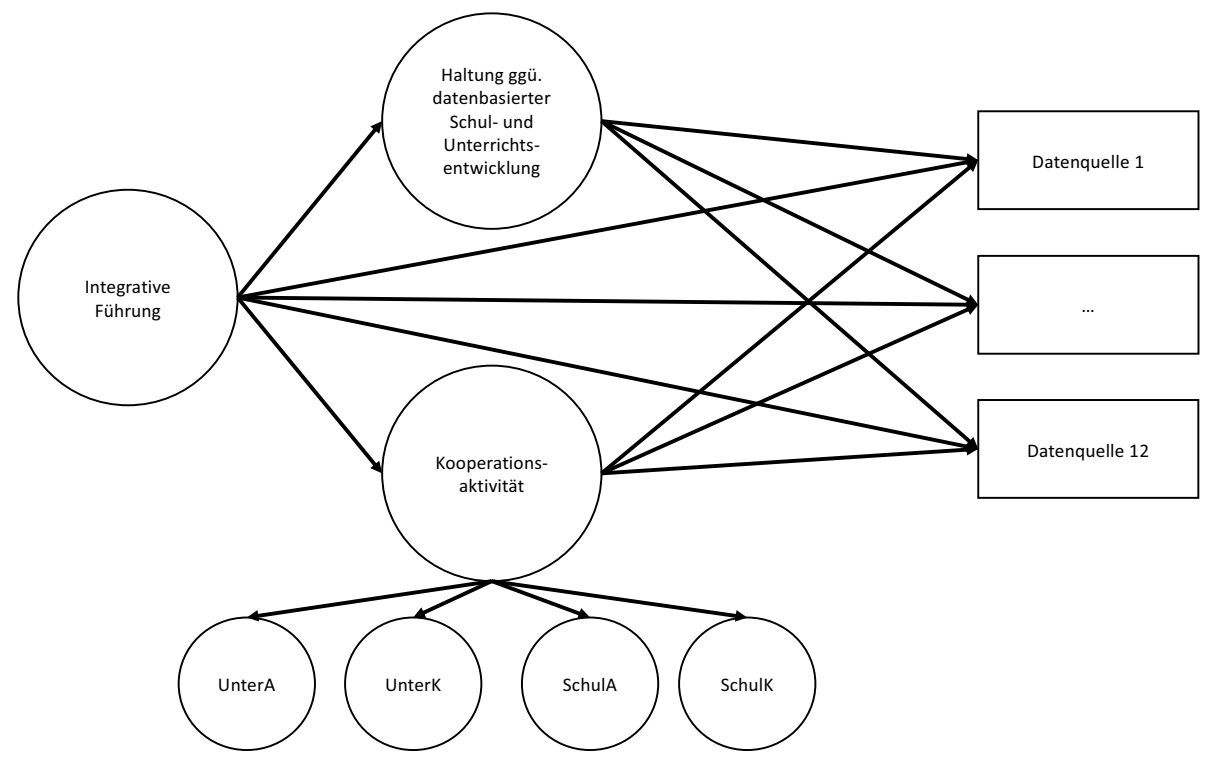

Anm.: UnterA = Unterrichtsbezogener Austausch, UnterK = Unterrichtsbezogene Kokonstruktion, SchulA = Schulbezogener Austausch, SchulK = Schulbezogene Kokonstruktion.

Quelle: eigene Darstellung 
Den größten direkten und statistisch signifikanten Zusammenhang hat integrative Führung mit der Nutzungshäufigkeit von Informationen über Unterrichtsausfälle/ Krankheitsstand, Befragungen von Schüler*innen und Eltern und schulinternen Evaluationen. Kleinere, aber signifikante Zusammenhänge bestehen zwischen dem Führungsverhalten und der Nutzung von Schüler*innenfehlzeiten und Schulinspektionsergebnissen. Für die Nutzung weiterer Datenquellen durch Lehrkräfte lassen sich keine signifikanten direkten Zusammenhänge mit dem Führungsverhalten der Schulleitung nachweisen.

Bei Einbezug der indirekten Effekte, die vermittelt über die Kooperationsaktivitäten und die Einstellungen der Lehrkräfte analysiert werden, wird der Zusammenhang zwischen integrativer Führung der Schulleitung und der Nutzungshäufigkeit aller abgefragten Daten- und Informationsquellen statistisch bedeutsam. Der größte Gesamteffekt ist dabei bei der Nutzung von schulinternen Evaluationen und Befragungen von Schüler*innen und Eltern zu beobachten. Der Zusammenhang mit der Nutzung von erreichten Abschlüssen des Abschlussjahrgangs, Noten einzelner Schüler*innen, Schulinspektionsergebnissen, Schüler*innenfehlzeiten sowie Unterrichtsausfall und Fachliteratur liegt mit Effektgrößen zwischen .32 und .43 im mittleren Bereich. Kleine, aber signifikante Gesamteffekte des Schulleitungshandelns sind weiterhin bei der Nutzung von Daten aus Vergleichsarbeiten, Schülerinnenfeedback zum Unterricht und bildungswissenschaftlichen Forschungsresultaten zu finden. Den kleinsten Effekt zeigt integratives Führungsverhalten bei der Nutzungshäufigkeit von allgemeinen Medien.

Weiterhin fällt auf, dass der Gesamteffekt vorwiegend über die Kooperationsaktivität der Lehrkräfte vermittelt zu sein scheint: Alle über die Kooperationsaktivität vermittelten Effekte von integrativer Führung sind hochsignifikant außer im Hinblick auf die Nutzung von Informationen zum Unterrichtsausfall und Krankenstand. Bei der Nutzung von Fachliteratur, bildungswissenschaftlichen Forschungsresultaten und allgemeinen Medien scheint darüber hinaus auch die Haltung der Lehrkräfte gegenüber datenbasierter Praxis den Effekt der Schulleitung zu vermitteln. Ein direkter Effekt des Führungshandelns auf die Datennutzung ließ sich bei diesen drei Datenquellen nicht nachweisen. 
Effekte von integrativer Führung auf die Datennutzung von Lehrkräften

Tab. 4: Direkte, indirekte und Gesamteffekte (beta $\beta$ ) von integrativer Führung auf die Nutzung von Evidenzquellen sowie die Varianzaufklärungen $R^{2}$

\begin{tabular}{|c|c|c|c|c|c|}
\hline \multirow[t]{2}{*}{ Daten und Informationsquellen } & \multicolumn{4}{|c|}{ Effekte integrativer Führung } & \multirow[b]{2}{*}{$R^{2}$} \\
\hline & direkt & $\begin{array}{c}\text { indirekt } \\
\text { Kooperation }\end{array}$ & $\begin{array}{l}\text { indirekt } \\
\text { Haltung }\end{array}$ & gesamt & \\
\hline $\begin{array}{l}\text { Erreichte Abschlüsse des Abschluss- } \\
\text { jahrgangs }\end{array}$ & .13 & $.23^{* *}$ & .00 & $.37^{* *}$ & $.11^{* *}$ \\
\hline Daten aus Vergleichsarbeiten & .04 & $.19 * *$ & -.01 & $.22^{* *}$ & $.06^{* *}$ \\
\hline $\begin{array}{l}\text { Ergebnisse zur Leistungsentwicklung/ } \\
\text { Noten einzelner Schüler*innen }\end{array}$ & .06 & $.23^{* *}$ & .03 & $.32^{* *}$ & $.10^{* *}$ \\
\hline $\begin{array}{l}\text { Schüler*innenfeedback zum Unter- } \\
\text { richt }\end{array}$ & -.04 & $.29 * *$ & .03 & $.28^{* *}$ & $.11^{* *}$ \\
\hline Ergebnisse aus der Schulinspektion & $.18^{*}$ & $.22^{* *}$ & .00 & $.41^{* *}$ & $.14^{* *}$ \\
\hline Schulinterne Evaluation & $.29 * *$ & $.26^{* *}$ & .03 & $.57 * *$ & $.20^{* *}$ \\
\hline Schüler*innenfehlzeiten & $.17^{*}$ & $.16^{* *}$ & .01 & $.35^{* *}$ & $.07^{* *}$ \\
\hline $\begin{array}{l}\text { Befragungen von Schüler*innen } \\
\text { und Eltern }\end{array}$ & $.30^{* *}$ & $.25^{* *}$ & .01 & $.56^{* *}$ & $.17^{* *}$ \\
\hline Unterrichtsausfall/Krankenstand & $.32 * *$ & .09 & .02 & $.43^{* *}$ & $.05^{* *}$ \\
\hline Fachliteratur & .08 & $.16^{* *}$ & $.09 * *$ & $.34 * *$ & $.12^{* *}$ \\
\hline $\begin{array}{l}\text { Bildungswissenschaftliche } \\
\text { Forschungsresultate }\end{array}$ & -.03 & $.19 * *$ & $.14^{* *}$ & $.30^{* *}$ & $.20^{* *}$ \\
\hline $\begin{array}{l}\text { Allgemeine Medien (z.B. Tages- } \\
\text { zeitung, Internet) }\end{array}$ & .02 & $.11^{* *}$ & $.03^{*}$ & $.16^{* *}$ & $.06^{* *}$ \\
\hline
\end{tabular}

Anm.: $\quad$ indirekt Kooperation $=$ über Kooperation vermittelte Effekte von integrativer Führung; indirekt Haltung = über Haltung der Lehrkräfte gegenüber datenbasierter Praxis vermittelte Effekte integrativer Führung; Signifikanzniveau: ${ }^{*} \mathrm{p}<.001,{ }^{\star} \mathrm{p}<.05$.

Quelle: eigene Berechnungen

\section{Diskussion}

Die Ergebnisse zeigen, dass die Nutzungshäufigkeit von Datenquellen durch Lehrkräfte von der Art und Komplexität des Mediums sowie vom unmittelbaren Bezug zur schulischen Praxis abhängt. Lehrkräfte gaben an, in ihrem Berufsalltag allgemeine Medien am häufigsten zu nutzen; dies kann an der besseren Verfügbarkeit und generell intensiveren Nutzung dieser Medien (z.B. Tageszeitungen) im Vergleich zu schulspezifischen Daten oder Ergebnissen der Bildungsforschung liegen. Weiterhin wurden Quellen mit einem direkten Bezug zu Schüler*innen oder zum Unterricht (z.B. Fehlzeiten, Noten), die häufig ohnehin an der Schule vorliegen und Lehrkräften zugänglich sind, besonders häufig genutzt. Möglicherweise sehen Lehrkräfte in die- 
sen Daten mehr handlungsleitende Informationen, z.B. für die Veränderung von Unterricht und Schule. Diese Daten werden bei gegebener Intention sogar teilweise direkt im Schulalttag durch die Lehrkräfte generiert (z. B. Schülerinnenfeedback). Sie sind somit weniger schwer zu verstehen als extern generierte Daten wie bildungswissenschaftliche Forschungsresultate, Daten aus Vergleichsarbeiten oder Schulinspektionsergebnisse, welche erst durch intensive Beschäftigung und Auseinandersetzung in konkrete Veränderungsbedarfe und Handlungen übersetzt und anschließend in schulische Prozesse integriert werden können.

Weiterhin zeigen die Ergebnisse, dass integrative Führung sowohl direkt als auch indirekt mit der Nutzungshäufigkeit zusammenhängt. Der direkte Zusammenhang lässt sich vor allem bei Daten beobachten, die interne schulische Abläufe betreffen (z. B. Unterrichtsausfall/Krankenstand) oder bewerten (z. B. Schulinspektionsergebnisse, schulinterne Evaluationen). Dabei fällt auf, dass diese Daten vergleichsweise weniger genutzt werden. Eine Erklärung dafür liegt darin, dass diese Quellen möglicherweise nicht direkt an Lehrkräfte, sondern zunächst an die Schulleitungen gerichtet sind und die Lehrkräfte zum Teil auch keinen direkten Zugriff darauf haben. Die Ergebnisse lassen damit die Vermutung zu, dass Schulleitungen mit einer integrativen Führung, z. B. durch Schaffung günstiger Rahmenbedingungen und Vorgaben, die Auseinandersetzung der Lehrkräfte mit diesen Datenquellen ermöglichen. Weiterhin deuten die Analysen auf eine deutlich vermittelnde Funktion der Kooperationsaktivität im Kollegium hin. Eine daraus resultierende Annahme könnte sein, dass Schulleitungen durch ein integratives Führungsverhalten das Kooperationsverhalten der Lehrkräfte stärken können, welches dazu beiträgt, dass mehr Datenquellen durch Lehrkräfte genutzt werden. Diese datengestützten Spekulationen lassen sich jedoch anhand der vorliegenden Querschnittsdaten hinsichtlich kausaler Beziehungen nicht weiter überprüfen.

Die vorliegende Studie weist einige Einschränkungen auf. Eine methodische Schwäche liegt im Querschnittsdesign und in der Stichprobeneigenschaft der Studie. Durch die einmalige Erhebung von Daten an ausschließlich weiterführenden Projektschulen sind Aussagen zur Vorhersage der Modellvariablen nur eingeschränkt möglich. Weiterhin ist $\mathrm{zu}$ beachten, dass die integrative Führung eine eher pragmatische Kombination transformationaler und instruktionaler Aspekte ist und noch einer theoretischen Begründung und genauerer Konzeptualisierung hinsichtlich spezifischer Verhaltenskomponenten bedarf. Schließlich wurden nur ausgewählte Mediatorvariablen untersucht; weitere Analysen können z. B. die Fähigkeit der Lehrkräfte zur datenbasierten Praxis sowie die wahrgenommene Qualität und Nutzbarkeit der Daten berücksichtigen (vgl. Schildkamp et al., 2017). Wie in vielen anderen Studien in diesem Forschungsfeld wurde Datennutzung durch Lehrkräfte unabhängig von kontextbezogenen, systemischen Anforderungen und Rahmenbedingungen betrachtet, sodass Aussagen über kausale Mechanismen und vermittelnde Prozesse nicht möglich sind. So kann beispielsweise die Aussage getroffen werden, dass Lehrkräfte angaben, 
allgemeine Medien am häufigsten in ihrem Berufsalltag zu nutzen. Ob und welche Prozesse dabei für eine effektive Weiterentwicklung ihres eigenen Unterrichts und für ihre Schul- und Unterrichtsentwicklung daraus resultieren, sollte in zukünftigen Studien spezifischer untersucht werden.

Die Studie erhärtet Hinweise, dass Aspekte des Schulleitungshandelns mit der Nutzungshäufigkeit von Daten- und Informationsquellen zusammenhängen. Das Kooperationsverhalten der Lehrkräfte, welches diese Zusammenhänge vermittelt, ist von enormer Bedeutsamkeit und daher besonders zu beachten. Die Fragen, welche konkreten Verhaltenskomponenten von Führung Kooperationsverhalten begünstigen und welche Mechanismen der Kooperation tatsächlich dazu führen, dass Lehrkräfte sich mit den Daten auseinandersetzen, sind wichtige Grundlagen für zukünftige Studien. Dazu sind Studien erforderlich, die kausale Effekte untersuchen können und auf dieser Grundlage auch Konsequenzen für die Bildungspraxis ermöglichen.

\section{Literatur und Internetquellen}

Aarons, G. A., Glisson, C., Hoagwood, K., Kelleher, K., Landsverk, J., Cafri, G., \& Research Network on Youth Mental Health. (2010). Psychometric Properties and U.S. National Norms of the Evidence-based Practice Attitude Scale (EBPAS). Psychological Assessment, 22 (2), 356-365. https://doi.org/10.1037/a0019188

Albiladi, W., Lasater, K., \& Bengtson, E. (2020). Date Use among Principals and Teachers: Divergent Paths or Common Ground. Journal of School Administration Research and Development, 5 (2). Zugriff am 07.01.2021. Verfügbar unter: https://www.ojed.org/index. php/JSARD/article/view/2240.

Altrichter, H. (2010). Schul- und Unterrichtsentwicklung durch Datenrückmeldung. In K. Maag Merki \& H. Altrichter (Hrsg.), Handbuch Neue Steuerung im Schulsystem (S. 219-254). Wiesbaden: VS. https://doi.org/10.1007/978-3-531-18942-0

Anderson, S., Leithwood, K., \& Strauss, T. (2010). Leading Data Use in Schools: Organizational Conditions and Practices at the School and District Levels. Leadership and Policy in Schools, 9 (3), 292-327. https://doi.org/10.1080/15700761003731492

Bass, B. M., \& Avolio, B. J. (1994). Transformational Leadership and Organizational Culture. International Journal of Public Administration, 17 (3-4), 541-554. https://doi. org/10.1080/01900699408524907

Bromme, R., Prenzel, M., \& Jäger, M. (2014). Empirische Bildungsforschung und evidenzbasierte Bildungspolitik. Eine Analyse von Anforderungen an die Darstellung, Interpretation und Rezeption empirischer Befunde. Zeitschrift für Erziehungswissenschaft, 17, 3-54. https://doi.org/ 10.1007/s11618-014-0514-5

Brown, C., \& Zhang, D. (2016). Is Engaging in Evidence-informed Practice in Education Rational? What Accounts for Discrepancies in Teachers' Attitudes towards Evidence Use and Actual Instances of Evidence Use in Schools? British Educational Research Journal, 42 (5), 780-801.

Coburn, C. E., \& Turner, E. O. (2011). Research on Data Use: A Framework and Analysis. Measurement: Interdisciplinary Research and Perspectives, 9 (4), 173-206. https://doi.or g/10.1080/15366367.2011.626729 
Habibe Ercan, Ulrike Hartmann, Dirk Richter, Jenny Kuschel \& Cornelia Gräsel

Datnow, A., \& Hubbard, L. (2016). Teacher Capacity for and Beliefs about Data-driven Decision Making: A Literature Review of International Research. Journal of Educational Change, 17 (1), 7-28. https://doi.org/10.1007/s10833-015-9264-2

Hallinger, P., Leithwood, K., \& Heck, R. H. (2010). Leadership: Instructional. In P. Peterson, E. Baker \& B. McGaw (Hrsg.), International Encyclopedia of Education (S. 18-25). Amsterdam: Elsevier.

Hartmann, U., Richter, D., \& Gräsel, C. (2020). Same Same But Different? Analysen zur Struktur kollegialer Kooperation unter Lehrkräften im Kontext von Schul- und Unterrichtsentwicklung. Unterrichtswissenschaft (2020). https://doi.org/10.1007/s42010020-00090-8

Heck, R. H., \& Hallinger, P. (2010). Testing a Longitudinal Model of Distributed Leadership Effects on School Improvement. The Leadership Quarterly, 21 (5), 867-885. https://doi. org/10.1016/j.leaqua.2010.07.013

Hinzke, J.-H., Gesang, J., \& Besa, K.-S. (2020). Zur Erschließung der Nutzung von Forschungsergebnissen durch Lehrpersonen. Forschungsrelevanz zwischen Theorie und Praxis. Zeitschrift für Erziehungswissenschaft, 23, 1303-1323. https://doi.org/10.1007/ s11618-020-00982-6

Ikemoto, G. S., \& Marsh, J. A. (2007). Cutting Through the "Data Driven" Mantra: Different Conceptions of Data-driven Decision Making. In P. A. Moss (Hrsg.), Evidence and Decision Making. 106 ${ }^{\text {th }}$ Yearbook of the National Society for the Study of Education (S. 105-131). Malden, MA: Blackwell.

Leithwood, K., \& Sun, J. (2012). The Nature and Effects of Transformational School Leadership: A Meta-analytic Review of Unpublished Research. Educational Administration Quarterly, 48 (3), 387-423. https://doi.org/10.1177/ 0013161X11436268

Lewin, J., \& Datnow, A. (2012). The Principal Role in Data-driven Decision Making: Using Case-study Data to Develop Multi-mediator Models of Educational Reform. School Effectiveness and School Improvement, 23 (2), 179-201. https://doi.org/10.1080/09243 453.2011.599394

Lüdtke, O., Trautwein, U., Kunter, M. \& Baumert, J. (2006). Analyse von Lernumwelten. Ansätze zur Bestimmung der Reliabilität und Übereinstimmung von Schülerwahrnehmungen. Zeitschrift für Pädagogische Psychologie, 20 (1/2), 85-96. https://doi. org/10.1024/1010-0652.20.12.85

Mandinach, E., \& Gummer, E. S. (2016). What Does It Mean for Teachers to Be Data Literate: Laying Out the Skills, Knowledge, and Dispositions. Teaching and Teacher Education, 60, 366-376. https://doi.org/10.1016/j.tate.2016.07.011

Marsh, J. A. (2012). Interventions Promoting Educators' Use of Data: Research Insights and Gaps. Teachers College Record, 114 (11), 1-47.

Marsh, H. W., Hau, K.-T., \& Wen, Z. (2004). In Search of Golden Rules: Comment on Hypothesis-Testing Approaches to Setting Cutoff Values for Fit Indexes and Dangers in Overgeneralizing Hu and Bentler's (1999) Findings. Structural Equation Modeling: A Multidisciplinary Journal, 11, 320-341. https://doi.org/10.1207/s15328007sem1103_2

Muthén, L. K., \& Muthén, B. O. (2006). Mplus User's Guide (4. Aufl.). Los Angeles, CA: Muthén \& Muthén.

Pietsch, M. (2014). Instruktionale Führung an Schulen in Hamburg. Hamburg macht Schule, $26(2), 24-27$.

Pietsch, M., \& Hosenfeld, I. (2017). Inspektionsbasierte Schul- und Unterrichtsentwicklung. Landau: Verlag Empirische Pädagogik.

Schildkamp, K., \& Ehren, M. C. M. (2013). From "Intuition"- to "Data"-based Decision Making in Dutch Secondary Schools? In K. Schildkamp, M. K. Lai \& L. M. Earl (Hrsg.), Data-based Decision Making in Education. Challenges and Opportunities (S. 49-67). Dordrecht: Springer. 
Schildkamp, K., \& Lai, M. K. (2013). Conclusions and a Data Use Framework. In K. Schildkamp, M. K. Lai \& L. M. Earl (Hrsg.), Data-based Decision Making in Education. Challenges and Opportunities (S. 177-191). Dordrecht: Springer.

Schildkamp, K., Poortman, C., Luyten, H., \& Ebbeler, J. (2017). Factors Promoting and Hindering Data-based Decision Making in Schools. School Effectiveness and School Improvement, 28 (2), 242-258. https://doi.org/10.1080/09243453.2016.1256901

Schmich, J., \& Schreiner, C. (Hrsg.). (2008). TALIS 2008. Schule als Lernumfeld und Arbeitsplatz. Erste Ergebnisse des internationalen Vergleichs. Graz: Leykam.

Stump, M., Zlatkin-Troitschanskaia, O., \& Mater, O. (2016). The Effects of Transformational Leadership on Teachers' Data Use. Journal for Educational Research Online, 8 (3), 8099.

Thiel, F., Hannover, B., \& Pant, H. A. (2014). Nutzung und Effekte zentraler Abschlussprüfungen und standardbasierter Schulleistungstests als Instrumente der Neuen Steuerung. Zeitschrift für Erziehungswissenschaft, 1, 3-6. https://doi.org/10.1007/s11618-0140482-9

Zlatkin-Troitschanskaia, O. (2016). Evidence-based Actions within the Multi-level System of Schools - Requirements, Processes, and Effects (EviS). Special Issue Editorial. Journal for Educational Research Online, 8, 5-13.

Zlatkin-Troitschanskaia, O., Zimmer, L., Mater, O., Laier, B., Koch, A. R., Binnewies, C., et al. (2016). Schulische und individuelle Einflussfaktoren auf das evidenzbasierte Handeln von Lehrkräften und Schulleitungen: eine mehrebenenanalytische Studie. In Bundesministerium für Bildung und Forschung (BMBF) (Hrsg.), Steuerung im Bildungssystem: Implementation und Wirkung neuer Steuerungsinstrumente im Schulwesen (S. 8-38). Berlin: BMBF. Zugriff am 07.01.2021. Verfügbar unter: https:// www.bmbf.de/upload_filestore/pub/Bildungsforschung_Band_43.pdf.

Habibe Ercan, geb. 1989, Wissenschaftliche Mitarbeiterin am Institut für Bildungsforschung in der School of Education an der Bergischen Universität Wuppertal.

E-Mail: ercan@uni-wuppertal.de

Cornelia Gräsel, Prof. Dr., geb. 1966, Professorin am Institut für Bildungsforschung in der School of Education an der Bergischen Universität Wuppertal.

E-Mail: graesel@uni-wuppertal.de

Korrespondenzadresse: Bergische Universität Wuppertal, Institut für Bildungsforschung, Abteilung Lehr-, Lern- und Unterrichtsforschung, Gaußstraße 20, 42119 Wuppertal

Ulrike Hartmann, Dr., geb. 1980, Wissenschaftliche Mitarbeiterin im Arbeitsbereich Schulisches Lehren und Lernen am DIPF | Leibniz-Institut für Bildungsforschung und Bildungsinformation.

E-Mail: u.hartmann@dipf.de

Korrespondenzadresse: DIPF | Leibniz-Institut für Bildungsforschung und Bildungsinformation, Abteilung Lehr- und Lernqualität in Bildungseinrichtungen, Rostocker Straße 6, 60323 Frankfurt a. M. 
| Habibe Ercan, Ulrike Hartmann, Dirk Richter, Jenny Kuschel \& Cornelia Gräsel

Dirk Richter, Prof. Dr., geb. 1980, Professor für Erziehungswissenschaftliche Bildungsforschung an der Universität Potsdam.

E-Mail: dirk.richter@uni-potsdam.de

Jenny Kuschel, geb. 1989, Akademische Mitarbeiterin an der Professur für Erziehungswissenschaftliche Bildungsforschung an der Universität Potsdam.

E-Mail: jkuschel@uni-potsdam.de

Korrespondenzadresse: Universität Potsdam, Professur für Erziehungswissenschaftliche Bildungsforschung, Karl-Liebknecht-Straße 24-25, 14476 Potsdam 